\title{
El malestar: codificación de la crisis española en Clavícula de Marta Sanz
}

\section{The Malaise: Codification of the Spanish Crisis in Clavícula by Marta Sanz}

OLGA BuczeK [olga.buczek@poczta.umcs.lublin.pl]
Uniwersytet Marii Curie-Skłodowskiej w Lublinie, Polonia

Mariola Pietrak [mariola.pietrak@mail.umcs.pl]

Uniwersytet Marii Curie-Skłodowskiej w Lublinie, Polonia

\begin{abstract}
RESUMEN
La literatura es siempre política (ideológica) en cuanto que se sitúa dentro de un espacio discursivo específico, en el seno de un estado de sociedad dado. La literatura codifica siempre la realidad social, pero la escritura responsable (monitoring) se orientará al conflicto, que siempre subyace a esa realidad, y a las voces disidentes, articulando un discurso alternativo. En Marta Sanz tal codificación se opera mediante el cuerpo. Es un proceso (in)consciente que pone de manifiesto las secuelas de la crisis en la sociedad española y, en general, del capitalismo en el individuo (sobre todo femenino). En el presente trabajo, indagamos en estos puntos dándole especial importancia a cómo se textualiza lo político en la obra de la escritora madrileña.
\end{abstract}

\section{Palabras clave}

Crisis española; sociocrítica; cuerpo; literatura; política; codificación; autobiografía; Marta Sanz

\begin{abstract}
Literature is always political (ideological) insofar as it is situated within a specific discursive space, within a given state of society. Literature always encodes social reality, but responsible writing (monitoring) will be oriented to the conflict, which always underlies that reality, and to dissident voices, articulating an alternative discourse. In Marta Sanz, such codification is operated through the body. It is an (un)conscious process that reveals the consequences of the crisis in Spanish society and, in general, of capitalism in the individual (especially female). This article deals with these questions, focusing on the importance of the textualization of the political in Sanz's work.
\end{abstract}

\section{KEYWORDS}

Spanish crisis; Sociocriticism; body, politics; literature; codification; autobiography; Marta Sanz

RECIBIDO 2021-05-10; ACEPTADO 2021-07-01 
¿Sólo la «literatura política» es literatura política o toda la literatura lo es?

¿Es el texto un cuerpo y el cuerpo, un texto?

Marta Sanz, No tan incendiario $(2014: 13,98)$.

\section{Introducción}

La crisis española del 2008, inconclusa aún, marcó profundamente la literatura del país. Desde hace una década, la crítica debate el marcado "giro político" en las artes, así como el fantasma de la "literatura comprometida" que nuevamente recorre a Europa. Con él, recobran actualidad la discusión sobre la responsabilidad del artista en la sociedad y una "literatura de intervención social” (Lluch-Prats 2017), el compromiso político del escritor y la capacidad de la literatura de crear un discurso disidente y transformador en pleno auge del capitalismo (Potok 2020; Baltar 2014; Becerra 2015). Surcan la bibliografía crítica preguntas tales como: “es posible una novela crítica disidente, contrahegemónica, de oposición, [...]- en un momento histórico en que el capitalismo muestra su rostro más totalizador, que incluso ha hegemonizado nuestro inconsciente?" (Becerra 2015: 7, 10-11). Si es así, “¿cómo se articula literariamente un discurso disidente?” (2015: 11).

Esta última pregunta, sobre todo, fija el horizonte de nuestro acercamiento a Marta Sanz y a la forma en que lo político se textualiza en su novela Clavícula (2017). No dejamos de lado, sin embargo, la discusión general. En la primera parte de este trabajo, abordamos la inevitable politización de la literatura siendo, como es, producto discursivo de un estado de sociedad dado, de un momento histórico específico. Compartimos esta postura con los teóricos del discurso y la teoría sociocrítica: Marc Angenot (2010) y M.-Pierrette Malcuzynski (1991, 2006, entre otros). En las siguientes partes, nos centramos en el rol del cuerpo, especialmente importante (o: en el que Sanz pone especial atención) en toda la obra de Sanz y, en especial, en Clavícula (Martín Huertas 2020). Como afirma la propia Sanz, "Clavícula nace de la percepción de que el cuerpo es un texto y el texto es un cuerpo" (Rovecchio 2017b). Por último, nos detenemos brevemente en la función del lenguaje y el corte autobiográfico que se inscriben en la labor contrahegemónica de la novela.

Marta Sanz (Madrid, 1967) es una de las escritoras más nombradas en la nómina de los "escritores comprometidos", junto con Belén Gopegui, Rafael Chirbes, Cristina Morales, Manuel Rivas o Javier Mestre. No es de extrañar, teniendo en cuenta que, en más de una ocasión, esta escritora y académica española manifestó su interés por cuestionar el orden imperante y ha establecido la resistencia como el objetivo de su vida laboral (p. ej. en "Intemperies/resistencias" 2018a).

\section{Texto}

¿Es posible una novela contrahegemónica en plena posmodernidad -la lógica cultural del capitalismo avanzado (Jameson) - cuando ya no quedan interferencias de otras ideologías de ningún signo, por lo cual su hegemonía y el control sobre la vida humana son ilimitados? (Becerra 2015: 8). En su célebre texto Teoría e historia de la producción ideológica (1990), Juan Carlos Rodríguez desvelaba la existencia de las primeras literaturas burguesas ya en el Renacimiento, cuando la matriz feudal era aún plenamente sólida, un par de siglos antes de su efectivo surgimiento o flo- 
recimiento. Por su parte, Marc Angenot (2010) recuerda que siempre coexisten, en un mismo espacio social, verdades contradictorias, periféricas, residuales o emergentes. Por lo general, están encubiertas (que no acalladas) bajo el velo de una ilusoria unidad de un estado determinado del discurso social, de lo pensable y de lo decible, esto es, de lo legítimo y lo legible de una época dada.

Sin embargo, en las zonas fronterizas -campos llenos de contradicciones- y en las épocas del des-orden social -como las crisis económicas o políticas- esas fuerzas opositoras y centrífugas se intensifican poniendo de manifiesto la verdadera naturaleza del espacio social: como un vasto rumor de los discursos sociales (alternativos, discordantes, transversos) en constante pugna por imponerse como dominante. El texto re-produce estas fricciones existentes entre los varios discursos y fuerzas sociales que concurren en una instancia social dada ${ }^{1}$.

Todos los teóricos, y también Marta Sanz (2018a), se ponen de acuerdo en que el escritor no escapa de su "ya-allî" del discurso social, ese estado determinado de la discursividad que le tocó vivir. Marta Sanz sigue en este aspecto al filósofo esloveno Slavoj Žižek y su concepto de la "ideología invisible: aquellas creencias y valores", según explica, "que tenemos tan naturalizados que ya no sentimos como parte de un constructo ideológico. La ideología invisible ya no se percibe como ideología, sino como sentido común”, y el "sentido común no se discute” (Sanz 2018a: 1).

Que sea indiscutible en una época no quiere decir que no sea inmodificable. La teoría sociocrítica insiste en toda una gama de procesos que ocurren entre el paso de la materia pretextual a la materia textual. El texto constituye un "dispositivo interdiscursivo e intertextual que absorbe y vuelve a poner de modo específico [...] y singular las representaciones de lo real presentes en el "ya-allí» del discurso social" (Robin y Angenot 1991: 77; cursiva nuestra). Resulta decisiva, en este sentido, la mediación del escritor: un sujeto creador afectado por el paso de sujeto ideológico a sujeto crítico. No se trata solo de las actitudes (in)conscientes en la selección de lo que (se) dice y qué no (se) puede decir, cómo (se) dice lo que es dicho y cómo (se) silencia lo que es silenciado. Se trata, sobre todo, de la postura deliberada que el sujeto creador adopte frente a ese barullo no acallado de los discursos cacofónicos y antagonistas, un posicionamiento responsable y atento a lo que debe ser transmitido o trabajado, lo que debe ser ficcionalizado ("mise en fiction"). Es lo que M.-Pierrette Malcuzynski denomina monitoring. En sus palabras:

Por monitoring se busca [...] interpelar la polifonía discursiva de manera comprensiva, trabajando, por una parte, las interacciones dialógicas intersubjetivas, las que definen las relaciones entre diversos sujetos, los cuales, a través de su práctica (textual), manipulan materiales discursivos igualmente diversos y no sólo lingüísticos y, por la otra, las relaciones dialécticas entre los sujetos y sus objetos. Distinguiendo [...] entre valor de cambio y valor de uso, el monitoring requiere de una teoría de la mediación que remita no sólo a la circulación de los discursos ["lo dado"], sino también a su producción ["lo creado"] y su materialización ["lo proyectado"], en el seno de un estado de sociedad dado, es decir, al sujeto productor. (Malcuzynski 2006: 24-25)

Gracias al monitoring, el sujeto creador marca su propia posición diferencial respecto de su tiempo proyectándolo en el texto. Lo "proyectado" se materializa en el texto dando cuenta

1 Según J. C. Rodríguez: "la dialéctica inscrita en los textos literarios [...] es la plasmación de un inconsciente ideológico que [...] 'nace' [...] directamente en el interior de las relaciones sociales mismas y desde ellas únicamente se segrega" (1990: 23). 
reflexivamente de la interacción generalizada entre lo cultural, lo intersubjetivo y todo factor histórico, sociopolítico, etc., que subyace a la producción y la recepción de los textos. La mediación del sujeto creador es crucial porque es él el umbral en que se negocian lo "dado" y lo "creado" (la textualización de una sociodiscursividad dada y, viceversa, el impacto del texto en el mundo social). Es donde, para Jacques Rancière, "ocurre la política": en el uso de la palabra que es crítica reflexiva del mundo común "y no solamente una voz que denota dolor" (2011: 34). Sobre esta apostilla de Rancière volveremos en breve.

La conciencia de estos procesos es muy patente en toda la obra de Marta Sanz. Ella marca su posición diferencial con un distanciamiento abierto respecto de la sociodiscursividad española tardocapitalista, pero también con ciertas alianzas textuales. En No tan incendiario (2014), declara su inconformidad y la convicción de que toda literatura debe ser política; en Monstruas y centauras (2018b: 9) aclara que lo es, porque es producto del metabolismo de la cultura "que se nos hace bola". De Bourdieu coge el "posicionamiento en el espacio de lo real", que "nos significa dentro de un campo, [...] un territorio acotado con unas leyes más o menos rígidas” (Sanz 2018a: 2; referencia a Bourdieu en el original). De Adrienne Rich, la "política de la ubicación” que enriquece esta postura y le agrega elementos cruciales de la geografía y el cuerpo (Sanz 2018a: 5). En la misma línea que Malcuzynski, Rich vincula cada afirmación a un cuerpo situado histórica y geográficamente, atravesado por la especificidad de la propia realidad social, étnica, de clase, económica y sexual ${ }^{2}$.

Por lo mismo, en "Intemperies/resistencias", Sanz ubica su escritura en el territorio acotado por su condición de española, mujer, escritora, de clase media expuesta al desempleo, entre otros (2018a: 5). Son las características que "me convierten", sostiene, "en la persona que soy y en la escritora que soy porque definen mis intereses y me dotan de unas herramientas y no de otras, de un idioma y no de otro" (2018a: 5). Este último es especialmente importante: no es solo un lenguaje, herramienta inofensiva, sino que es una “jaula” -" jaula del lenguaje”, en sus palabras-, ya que el lenguaje es portador de "una visión del mundo que no [se puede] evitar y de otra contra la que [ella se construye]" (2018a: 5). Como confiesa, es una "intemperie [...] que, ideológicamente, neutraliza desde un primer momento la posibilidad de que la literatura actúe como herramienta de denuncia y contrapeso, como resistencia, frente a esa ideología dominante" (Sanz 2018a: 3).

Por eso, precisamente, su primer movimiento es desplazar el peso de la enunciación del ámbito del lenguaje al cuerpo. El segundo movimiento le hará incursionar en el ámbito de la experiencia y la autobiografía.

\section{Cuerpo}

Para Sanz, el cuerpo es, en primer lugar, como decíamos, la posibilidad de un discurso contrahegemónico, de resistencia a un discurso dominado por varones. Si bien no reniega de los maestros de la cultura occidental (Ovidio, Lorca, Dostoievski), insiste en la necesidad de un discurso alternativo que sea capaz de describir la realidad de las "minorías” no hegemónicas. Por eso, todos sus

2 Véase Blood, Bread, and Poetry (Londres, The Women's Press, 1985), de A. Rich; asimismo, los conceptos de "locus enuntiationis" de Gayatri Spivak y "situatedness" de Dona Haraway. 
libros "utilizan un lenguaje del cuerpo que regresa al cuerpo a la vez que conciben la anatomía como una forma del lenguaje" (Sanz 2018a: 5). El "lenguaje del cuerpo", que postula, no solo reconfigura y resignifica términos ya existentes en el lenguaje institucional (Luce Irigaray), sino que también proporciona nuevos sentidos para verbalizar la experiencia femenina (Hélène Cixous). El cuerpo se convierte en un lienzo en que se habla de la experiencia común con el dolor, la enfermedad, la queja ${ }^{3}$.

Resuena aquí la crítica de Sanz a toda una tradición que repetía tras Aristóteles que solo el hombre posee la capacidad política pues "posee la palabra que pone en común lo justo y lo injusto mientras que el animal solamente posee la voz que señala placer y pena" (Rancière 2011: 34). En la construcción del sujeto moderno las mujeres quedaron de lado de la naturaleza, pero su "voz de dolor" cobra ahora una nueva fuerza política ${ }^{4}$.

En segundo lugar, Marta Sanz percibe el cuerpo como la cartografía de las contradicciones con el discurso tardocapitalista, somatizaciones de los desacuerdos declarados y de los que ni siquiera se es consciente.

Toda la novela se tiñe de la sensación de un gran malestar. Ese malestar rechina a lo largo de la lectura y obliga al lector a co-sentir y pensar. Clavícula es, como dice Martín Huertas (2020: 77), uno de estos "textos que duelen", textos que molestan, que llevan a la comunión de las sensaciones (vid. también Sanz 2014: 82). El dolor — tenaz e irrevocable- no es una metáfora, sino una realidad tangible, somatización pura del desacuerdo, físico, corpóreo, con el pathos posmoderno que lo hegemoniza todo. Se vincula explícitamente con la crisis y la codificación, por y en el texto, del malestar común que esta primera provoca. Cuando en una de las tantas consultas la doctora le pregunta “¿A qué le tienes miedo?”, ella, la narradora protagonista, responde: “A estar enferma. A no poder trabajar":

Entre hipidos e interrogaciones le repito a la médica esa respuesta que me ha dejado asombrada “Tengo miedo a no poder trabajar". [...] Enfermo del miedo a enfermar y del miedo a no poder enfermar. A que se hunda el mundo. A que la enfermedad se relacione con la imposibilidad de pagar las facturas. (Sanz 2020: 54)

Mi dolor es una letra que se escribe cuando tengo miedo de no poder pagar las facturas o subvencionarme una vejez sin olor a vieja. (Sanz 2020: 69)

La novela narra la experiencia de una mujer que, en un momento dado, a sus 50 años más o menos, empieza a sentir un dolor repentino, intenso y muy molesto en la clavícula, un hueso que, según el dictamen médico, en sí, no lesionado, no puede doler. El dolor que sufre la narradora -un dolor sin etiología clara, descrito como "una garrapata que atenaza el corazón" (Sanz 2020: 34)-, no se deja desligar del malestar general de su ser. Al contrario, debe entenderse como su portavoz, muy elocuente aunque estancado, por el momento, en el intento de encontrar un lenguaje propio. El problema de la dificultad de expresar verbalmente el dolor físico fue tratado ya en estudios tan importantes como The Body in Pain de Elaine Scarry (1987). En la novela de Sanz se aborda

3 Para este enfoque, vid. Kaiser Moro (2018), por ejemplo.

4 En este sentido, la narrativa de Sanz recoge la larga tradición de la écriture-femme promovida por el feminismo de segunda ola, retomada por los feminismos posteriores (de Sara Ahmed o Rosi Braidotti, entre muchas otras). Puede consultarse Béatrice Didier, L'écriture-femme. Presses Universitaires de France - PUF, 1981. 
desde la posible exuberancia verbal contrastada con la preocupación por la escasa operatividad comunicativa del lenguaje para las sensaciones de una mujer:

¿Han probado a buscar las palabras exactas para describir ese dolor, convertido en síntoma, que ayuda a los médicos a diagnosticar? [...] rebuscas dentro del baúl de palabras arrumbado en tu memoria. "Mi dolor es..." Nudo, corbata, pajarita, calambre, ausencia, hueco invertido [...]. Conozco bien el lenguaje y sus figuras retóricas. Pero soy tan imprecisa. No puedo explicarme [...]. (Sanz 2020: 61)

Sin embargo, es precisamente en esa brecha entre sentir el dolor y no poder contarlo donde nace la posibilidad de un lenguaje capaz de comunicar no solo la subjetividad más visceral del sujeto femenino, sino también su intuitiva resistencia frente a los discursos que lo atraviesan. Nombrar lo que antes carecía de nombre es un acto de creación y dominación; nombrar el dolor sería, por analogía, someterlo al dominio de la razón con sus estructuras vertebradas por convenciones sociales, etiquetas y prejuicios. Sucumbir a la tentación de ponerle un nombre a su malestar a cambio de un alivio y una promesa vaga de sintonizar con los demás, supondría, en última instancia, conferir su frágil materia a las economías afectivas del tardocapitalismo, enfocadas a comercializar remedios a toda indisposición que no encaje en la sociedad de la felicidad obligada (Ahmed 2019):

Puede que mi padre se enerve con mi sufrimiento o a causa de la intuición de que han conseguido convertirme en una víctima que necesita comprar justo eso que necesita y puede ayudarle a ser feliz. A paliar su dolor. Lo primero que necesito urgentemente es ponerle un nombre a lo que me pasa y, con el nombre, sentirme parte de algo. Busco mi grupo, mi target, mi gimnasio ideal, mi dieta sana, mi comunidad. (Sanz 2020: 146)

En el mundo organizado según las pautas neoliberales de la felicidad impuesta, se tiende a erradicar del imaginario colectivo cualquier referencia a lo político, estigmatizado como conflictivo, contraproducente e "ideológico" (Ahmed 2019: 15). Sus estructuras sociales se apoyan en la idea de colaboración, creatividad y optimismo, y rechazan formas específicas de malestar o emociones "negativas" (la vergüenza, el miedo...). Sentir malestar y comunicarlo con un lenguaje que emane del cuerpo femenino es subvertir el ideario neoliberal y capitalista, sumamente masculino, orientado a la productividad y la competitividad.

La escritora elige el acto de queja para comunicar su desacuerdo con los discursos dominantes que relegan el malestar femenino al reducto de lo voluble, insensato, inventado o invisible. Las mujeres aquejadas de dolor con más frecuencia que los hombres corren el riesgo de ser tildadas de hipocondríacas, impostoras, incluso locas. Sanz reivindica su derecho al malestar en el mundo que quiere ver en ellas madres devotas, trabajadoras sumisas u objetos sexuales que dan lo mejor de sí sin esperar ningún tipo de recompensa: "Asumo el discurso de los hipocondríacos y me ciño a la mirada de lo que los demás esperan de mí. Pero hoy me rebelo. No soy una hipocondríaca. No estoy deprimida. Tengo un dolor. Una enfermedad. Lo reivindico. Me quejo" (Sanz 2020: 86).

El sentido político de la queja queda, también, reflejado por la autora en la entrevista a Pliego Suelto del 18/11/2017: 
Uno de los temas fundamentales de Clavícula [...] es el de la legitimidad de la expresión del dolor. [...] [Y] decirte que no te quejes porque otros están peor que tú, en mi opinión, constituye una estrategia de desactivación política. Es como ponerle palos en la rueda a la conciencia crítica para hacerte sentir un miserable. (Rovecchio 2017b)

La queja pasa a ser, en consecuencia, una realización práctica del lema feminista "Lo personal es político", y, en palabras de la propia autora, "un acto de valentía y solidaridad en un mundo de emoticonos sonrientes y gente vestida de rosa" (Rovecchio 2017b).

La enfermedad constituye otra de las vías, al lado del malestar general y la queja, para codificar el capitalismo en España, y más precisamente, la crisis de funcionamiento, su propio agotamiento o insuficiencia. Se dice en el libro:

Las mujeres padecemos enfermedades misteriosas, enfermedades que se colocan en el límite de lo psiquiátrico y lo muscular, a través de lo neurológico, porque somos más sensibles al ruido, a la deformación, y nos resistimos a las inercias de nuestra forma de vida. Sin darnos cuenta, nos resistimos al neoliberalismo somatizándolo y nuestras somatizaciones se transforman en un interesado misterio de la ciencia. (Sanz 2020: 134-135; cursiva nuestra)

Son enfermedades que se escapan a la academia del lenguaje médico positivista, heteropatriarcal. En un sistema enfocado en el alto rendimiento y competitividad no hay lugar para debilidad y reposo. La contradicción del "sexo débil" -y por ello siempre amenazado en primer lugar de desempleo o precariedad laboral, y más en enfermedad o menopausia- encuentra expresión en el texto en toda una serie de enfermedades "misteriosas": la endometriosis, la fibromialgia, el insomnio, la costocondritis, y un largo etcétera. Estas no solo escapan al lenguaje, sino también a la cura rápida que devuelve el sujeto al engranaje capitalista de competitividad y productividad. La narradora no calla su situación de la precariedad; al contrario, la comparte con otras muchas mujeres, amigas, familiares, lectoras, creando una peculiar comunidad que se reconoce en su vulnerabilidad (vid. vida precaria de Judith Butler). "Somos tantas locas. Tantas", afirma Sanz en la novela (2020: 96), y, en otro momento, ironizando: "Qué hijas de puta somos las enfermas imaginarias" (2020: 66). En esta comunidad tienen cabida también los varones, al menos aquellos conscientes de su propia vulnerabilidad ante el sistema capitalista: "descubro - dice la narradora- que casi todo el mundo ha pasado por lo mismo que yo: Juan, Nuria, Verónica, José, Mercedes, Mabel... Echo la cuenta y me salen más mujeres. No creo que sea una cuestión de debilidad, sino de incremento de la presión" (Sanz 2020: 95).

Contar la condición vulnerable de una mujer en crisis (laboral y personal) es, sin duda, el gran tema de la novela. El cuerpo de la narradora de Clavícula somatiza su preocupación por la situación laboral, marcada por la irregularidad de su salario y el desempleo de su pareja, inscribe en sus tejidos el miedo a la pobreza que castiga, sobre todo, a las mujeres. La ansiedad, esa "patología del capitalismo avanzado", como leemos en la contracubierta de la edición citada, empuja a la escritora a dedicarse intensamente a su trabajo, sin permitirse un descanso. En consecuencia, se autoexplota ante la amenaza de precariedad, sin embargo, en varios momentos advierte su propia inmersión en el discurso capitalista de productividad que coloniza todos los ámbitos de su vida. Dice: 
La vida consiste en trabajar todo el día y culparse por esos momentos en que no se está trabajando. Hay una desproporción, un inmenso desajuste entre esfuerzo y remuneración que me obliga a multiplicar el número de mis trabajos para poder mantener mi nivelito de vida. (Sanz 2020: 69)

En otra ocasión, la narradora afirma, medio irónica: "sufro cuando siento que se me gasta la capacidad de trabajo imprescindible para la autoexplotación, porque en la autoexplotación reside el germen de mi felicidad" (2020: 64). Sus palabras llaman la atención del lector sobre la "ideología invisible" (término de Žižek) del capitalismo neoliberal: hemos racionalizado y naturalizado el rendimiento profesional hasta perder de vista su dimensión ideológica y convertirlo en sentido común. El filósofo surcoreano Byung-Chul Han lo expresa en su crítica del capitalismo neoliberal. En su famoso Psicopolítica. Neoliberalismo y nuevas técnicas de poder (2014) sostiene que, en la sociedad actual, de la que han desaparecido los mecanismos de esclavitud explícita, el individuo se somete al entramado de la dominación voluntariamente, seducido por el poder de la llamada psicopolítica. Como afirma Baratas Correo,

en el sistema neoliberal el sujeto participa en los procesos de autoexplotación en su búsqueda constante de rendimiento -o de productividad-, por lo que el capital se sirve del individuo, convirtiendo la libertad individual en libertad de capital. Y en esa relación de autoexplotación individual, la lucha de clases se transforma en una lucha personal, interna. Por otro lado, y de manera inconsciente, el individuo manifiesta culpabilidad hacia un capital que eleva como sagrado y ante el que siempre está en deuda, renunciando con ello a toda acción libre [...]. (2017: 885)

\section{Autobiografía}

Tal "sociedad de rendimiento" (Han 2012, 2014) es otro de los obstáculos, junto con el lenguaje opresor, para una escritura responsable, postulada por Malcuzynski y Sanz. En pos de la libertad del capital, el sujeto de rendimiento se priva de su propia libertad auto-oprimiéndose, convirtiéndose en explotador para sí mismo (Han 2012: 20). Por su parte, el lenguaje es portador de las normas sociales que conforman al sujeto, proporcionándole el marco y el punto de referencia para su ser, aunque, al menos para Judith Butler, no de una manera determinista (2009: 37). Sin embargo, el mismo instrumento que Sanz elige para eludir las “jaulas” del lenguaje y el neoliberalismo, se muestra así mismo problemático. Como observa la filósofa estadounidense, el cuerpo "no puede ser capturado por una narración total, no solo porque ese cuerpo tiene una historia formativa que es irrecuperable para la reflexión, sino porque el modo en que nos forman las relaciones primarias produce una opacidad ineludible en nuestra autocomprensión" (Butler 2009: 35).

Esta limitación básica no invalida el cuerpo como vía para codificar el mundo social, ni exime de la responsabilidad de dar cuenta de sí mismo, de contar la propia experiencia encarnada, de compartirla. Al contrario. Para Butler, cada sujeto tiene la obligación ética de producirse a sí mismo aun dentro de un "campo facilitador y limitante de coacciones", y este obrar dentro del campo del poder siempre constituirá un acto de poder, una agencia (2009: 33, 169). Para Sanz, esta obligación es más urgente aún en el contexto de la acuciante crisis que ha sufrido (sufre) España. Como confiesa en la entrevista a Pliego Suelto del 11/11/2017, ella siempre, y sobre todo en 
Clavícula, escribe "de lo que me duele física y socialmente. Relaciono mi dolor corporal con mi angustia laboral y conecto lo uno y lo otro con mi género para hacer un dibujo del miedo y de la fragilidad propia y ajena. [...] el lenguaje sirve para articular experiencias comunes" (Rovecchio 2017a). No obstante, en la realidad española a partir del 2008, la responsabilidad ética es pensar la violencia material y simbólica del sistema capitalista y sus secuelas en la sociedad actual, hostigada por el desempleo avasallador y deshumanizante. En la misma entrevista a Pliego Suelto, declara explícitamente: "Necesitamos vínculos fuertes - políticos y afectivos- en unos momentos en que todos los trabajadores han perdido derechos muy deprisa en todos los ámbitos laborales" (Rovecchio 2017a). Teniendo en cuenta que las mujeres están más expuestas a la precariedad laboral, su interés se centra principalmente en ellas.

La experiencia femenina le interesa también por otro motivo: constituye el espéculo de su propia experiencia, la imagen especular de su propio desnudo ${ }^{5}$. Como, parafraseando a Butler (2009), somos siempre parcialmente opacos para nosotros mismos, un modo de superar esa opacidad es el autorretrato o la autobiografía. Ambos equivalen así, para Sanz, a una posibilidad de reconquistar el espacio de su propio cuerpo:

Escribí [...] Clavícula porque me interesa hablar de mujeres desnudas, del desnudo de las mujeres, de su cuerpo. Y yo soy una mujer que escribe, y el procedimiento que tengo para desnudarme es mirarme en el espejo, hacer un ejercicio de introspección, contrastar mi cuerpo con el de otras mujeres y visibilizar nuestros dolores, tabúes, enfermedades, nuestro peculiar sentido del humor. (Rovecchio 2017a)

La autobiografía se revela como un modo de asir fragmentos del cuerpo, sus sombras y reflejos, y en esta fragmentariedad componer — como en el arte vitral- un relato de sí mismo, aún incompleto. El texto también es como ese cuerpo fragmentado, roto, fracturado con géneros o registros mezclados, su propia estructura fragmentaria, o fotos que de repente cortan el cuerpo lingüístico. Esas fotos de los pies autorales, según la declaración de Sanz, contribuyen tanto a la consagración de la realidad, del yo autobiográfico, como a la "fotogenia de los tabúes", que rodean los cuerpos de las mujeres, sobre todo las menopáusicas, los "cuerpos del trabajo" opuestos a los cuerpos mercantilizados del neoliberalismo (Rovecchio 2017a).

El cuerpo como texto y el texto como cuerpo, ambos rotos y dolidos, se ensamblan para escarbar en la realidad eludiendo la opresión y la insuficiencia del lenguaje, para volver menos opaco el cuerpo y dejarlo hablar. Como dice Marguerite Duras, cuyas palabras evoca Sanz en el epígrafe a Clavícula, "Uno se encarniza. No se puede escribir sin la fuerza del cuerpo" (Sanz 2020: 9).

Tal ruptura obliga al escritor y al lector a pensarse, pensar la vida propia, ajena, la vida en común: interpela al yo, al tú, a un nosotros. De hecho, como sostiene Martín Huertas, la autobiografía no se focaliza en el yo que escribe, sino que vuelve su mirada al lector, buscando incomodarlo con esta mirada, interrogarlo, perturbar (2020: 75). Sanz no busca narración total, ni tampoco una totalidad del cuerpo. Al contrario, la ruptura es premeditada, usada a conciencia para provocar el diálogo consigo mismo y con el otro. El lenguaje en sí es incapaz de conseguirlo, pero el lenguaje de las experiencias y el cuerpo es más eficaz. No reniega del yo: parte de él casi siempre, así como 
de sus propias experiencias personales y corporales. El punto de llegada es, como decíamos antes, articular las experiencias comunes, "la comunicación entre los seres humanos", hacer que "la literatura sea conversación” (Rovecchio 2017a).

\section{Conclusiones}

Nos preguntábamos al principio si es posible una novela disidente, contrahegemónica en el auge del capitalismo. No solo es posible, sino que es la esencia de una buena parte de la literatura, aquella literatura responsable atenta a la conflictividad social, a cierta "inquietud de época", "malestar cosmológico y hondísimo" (Sanz 2020: 53): es, en otras palabras, el "efecto colateral” del monitoring. Para Sanz, particularmente en las épocas como la crisis española, de la privación de derechos y la deshumanización, la responsabilidad ética del escritor es captar la violencia material y simbólica sufrida en un plano individual y llevar la experiencia al plano de las experiencias comunes.

El "cómo" se codificará esta disidencia en el texto depende ya de las propias condiciones materiales de la escritora (o escritor). Marta Sanz, siendo escritora, mujer de una edad que la acerca ya a la fase pos-productiva del capitalismo, elabora sus propias formas de articular su desacuerdo. Consciente de cómo funciona el espacio sociodiscursivo, la ideología y el lenguaje como su portador, recurre al cuerpo; pero no deja de lado el lenguaje, el que constantemente desafía. El cuerpo es el principal dispositivo de enunciación y critica, y ante sus limitaciones lo será también la autobiografía. Esta última constituye, por su parte, un efecto inevitable del monitoring:

Cuando escribo -cuando escribimos- no podemos olvidarnos de cuáles son nuestras condiciones materiales. Por eso pienso que todos los textos son autobiográficos y a veces la máscara [...] Me interesa más la pipa que la pipa que no es una pipa. La autobiografía es la consagración de la realidad [...] La escritura araña la entropía como una cucharilla de café el muro de la prisión. Amputa miembros. Identifica - para sanarlas- las lacras de la enfermedad. Es un escáner. (Sanz 2020: 50)

Lo que activa el monitoring en caso de Sanz es este conflicto interno que siente la narradora ante la precariedad laboral de las mujeres y artistas, y ante el desempleo de su marido y muchos otros como él. Es lo que la lleva, y con ella a los lectores, a ocupar una posición diferencial respecto del espacio socio-discursivo de la España tardocapitalista, sumergida en plena crisis. El malestar, la enfermedad, la queja... no solo somatizan su inconformidad con el discurso dominante y con su propio inconsciente ideológico, estructura o habitus hecho a medida de la doxa, del sentido común. Constituyen también una forma de textualizar dichas contradicciones, muy eficiente teniendo en cuenta que la emotividad y la afectividad se muestran escurridizas al lenguaje neoliberal: como dice Sara Ahmed (2019), vivimos el imperativo de ser felices.

Este alfabeto corporal hecho de las experiencias afectivas y corporales, somatizaciones, sensaciones, emociones, cumple con la larga ya tradición de la escritura femenina al tiempo que desafía otra -más larga aún- tradición que denigraba el cuerpo frente al logos-mente-cultura. Recordemos con Rancière (2011) que, para esta tradición, solo el que tenía voz, hacía política. Pero el mismo Rancière sostiene también que la política no hace referencia al puro ejercicio del poder o la lucha por este. "Es", dice, "la configuración de un espacio específico, el recorte de una esfera 
particular de experiencia", a lo que agrega "el conflicto [...] sobre la existencia de este espacio" y también "de los sujetos como provistos con la capacidad de una palabra común" (2011:33-34). El cuerpo en Clavícula se configura como este espacio, precisamente, donde se negocia la experiencia común a partir de la experiencia personal. Y se hace de forma tal que se desautomatiza lo que tenemos naturalizado volviendo posible el desarrollo de una mirada crítica sobre lo dado.

\section{Referencias bibliográficas}

Ahmed, S. (2019). La promesa de la felicidad. Una crítica cultural al imperativo de la alegría. Buenos Aires: Caja Negra.

Angenot, M. (2010). El discurso social. Los límites históricos de lo pensable y lo decible. Buenos Aires: Siglo XXI Editores.

Baltar, E. (2014). El fin de la literatura: ¿Hacia una politización de la cultura? Ábaco, 2, 82, 51-56.

Baratas Correo, A. M. (2017). Reseña de Han, Byung-Chul: Psicopolítica. Neoliberalismo y nuevas técnicas de poder. Política y Sociedad, 54, 3, 881-883.

Becerra Mayor, D. (2015). Introducción. In D. Becerra Mayor (coord.), Convocando al fantasma. Novela crítica en la España actual (pp. 7-24). Ciempozuelos: Tierradenadie Ediciones.

Butler, J. (2009). Dar cuenta de sí mismo. Violencia ética y responsabilidad. Buenos Aires: Amorrortu.

Han, B.-Ch. (2012). La sociedad del cansancio. Barcelona: Herder Editorial.

. (2014). Psicopolítica. Neoliberalismo y nuevas técnicas de poder. Barcelona: Herder Editorial.

Kaiser Moro, A. (2018). El derecho al aullido: corporalidad y lenguaje en Clavícula (Sanz, 2017). Feminismo/s 31, 189-203.

Lluch-Prats, J. (2017). Escritores españoles ante la crisis: propuestas de una literatura de intervención social. HispanismeS, 9, 4-16.

Malcuzynski, M.-P. (1991). Sociocríticas - prácticas textuales - cultura de fronteras. Amsterdam, Atlanta: GA. Rodopi.

. (2006). Yo no es un O/otro. Acta Poética, 27, 1, 19-44.

Martín Huertas, C. (2020). "Digo carne": La repolitización del cuerpo femenino en la narrativa de Marta Sanz. Studia Romanica Posnaniensia, 47, 1, 67-80.

Potok, M. (2020). Mujer y economía. La "narrativa de la crisis" desde la perspectiva de género. Studia Romanica Posnaniensia, 47, 1, 93-105.

Rancière, J. (2011). El malestar en la estética. Buenos Aires: Capital Intelectual.

Robin, R.; \& Angenot, M. (1991). La inscripción del discurso social en el texto literario. In M.-P. Malcuzynski (Ed.), Sociocríticas - prácticas textuales - cultura de fronteras (pp. 51-79). Amsterdam, Atlanta: GA. Rodopi.

Rodríguez, J. C. ([1974] 1990). Teoría e historia de la producción ideológica. 1. Las primeras literaturas burguesas (s. XVI). Madrid: Akal.

Rovecchio Antón, L. (2017a). Marta Sanz: "Me interesa una autobiografía que desdiga el tópico de la vanidad”. Pliego Suelto, 11/11/2017. http://www.pliegosuelto.com/?p=24161

_. (2017b). Marta Sanz: "Clavícula nace de la percepción de que el cuerpo es un texto y el texto es un cuerpo”. Pliego Suelto, 18/11/2017. http://www.pliegosuelto.com/?p=24219 
Sanz, M. (2014). No tan incendiario. Cáceres: Periférica.

. (2018a). Intemperies/resistencias. Olivar, 18, 27, 1-8.

. (2018b). Monstruas y centauras. Nuevos lenguajes del feminismo. Barcelona: Anagrama.

. (2020 [2017]). Clavícula. Barcelona: Anagrama.

Scarry, E. (1987). The Body in Pain: The Making and Unmaking of the World. London: Oxford University Press. 